\title{
Paediatric intraocular lens implants: accuracy of lens power calculations
}

MK O'Gallagher', MA Lagan', CP Mulholland'1, M Parker ${ }^{2}$, G McGinnity ${ }^{1}$ and EM McLoone ${ }^{1}$

\begin{abstract}
Purpose This study aims to evaluate the accuracy of lens prediction formulae on a paediatric population.

Methods A retrospective case-note review was undertaken of patients under 8 years old who underwent cataract surgery with primary lens implantation in a regional referral centre for paediatric ophthalmology, excluding those whose procedure was secondary to trauma.

Biometric and refractive data were analysed for 43 eyes, including prediction errors (PE). Statistical measures used included mean absolute error (MAE), median absolute error (MedAE), Student's $t$-test and Lin's correlation coefficient.

Results The mean PE using the SRK-II formula was +0.96 $\mathrm{D}$ (range - 2.47D to +2.41 D, SD 1.33 D, MAE 1.38 D, MedAE 1.55, $n=15)$. The mean PE was smaller using SRK/ $\mathrm{T}$ (-0.18 $\mathrm{D}$, range $-3.25 \mathrm{D}$ to $+3.95 \mathrm{D}$, SD $1.70 \mathrm{D}$, MAE $1.30 \mathrm{D}$, MedAE 1.24, $n=27$ ). We performed an analysis of the biometry data using four different formula (Hoffer $Q$, Holladay 1, SRK-II and SRK/T). Hoffer Q showed a smaller MedAE than other formulae but also a myopic bias. Conclusion Our clinical data suggest SRK/T was more accurate in predicting postoperative refraction in this cohort of paediatric patients undergoing cataract surgery. Hoffer $Q$ may have improved accuracy further.

Eye (2016) 30, 1215-1220; doi:10.1038/eye.2016.163; published online 29 July 2016
\end{abstract}

\section{Introduction}

There is increasing acceptance of the practice of intraocular lens (IOL) implantation in children undergoing cataract surgery. ${ }^{1,2}$ Accurate prediction of post-operative refraction is required in order to avoid amblyogenic refractive errors, anisometropia and to allow refractive targeting to take into account the future growth of the eye. Lens prediction formulae in common usage today are based on theoretical models or on regression from normative data from adult eyes. These formulae may not be as accurately applied to the paediatric range of axial lengths. ${ }^{3}$ In this study, we report the accuracy of adult lens prediction formulae when used on a paediatric population.

\section{Materials and methods}

A retrospective case-note review was performed in a regional tertiary referral centre. Patients were identified by searching admissions data and computerised theatre logbooks. Patients under the age of 8 years undergoing cataract surgery with IOL implantation between 1996 and 2008 were included in this study. Patients over 8 years of age and those undergoing lensectomy as part of repair of ocular trauma were excluded.

Forty-six patients (67 eyes) were identified. The following data were recorded: age at the time of surgery, axial length, pre-operative keratometry measurements, lens prediction formula used, lens implant type used and dioptric power of implant. The intended postoperative refraction, as calculated using the lens prediction formula, and the actual post-operative refraction at three months post-operatively were also recorded. This enabled a prediction error (PE) to be calculated ( $\mathrm{PE}=$ actual post-operative refraction-predicted refraction).

Axial lengths were measured by the surgeon on the operating table using contact A-scan ultrasonography (US-800 or US-1800, NIDEK, Aichi, Japan) and keratometry was measured using a handheld automated keratometer (KM-500, NIDEK, Aichi, Japan). The exceptions to this were five patients (eight eyes) whose axial lengths and keratometry were recorded using IOL Master (IOL Master, Carl Zeiss-Meditec,
${ }^{1}$ Department of Ophthalmology, Eye and Ear Clinic Royal Victoria Hospital, Belfast, UK

${ }^{2}$ Northern Ireland Clinical Research Support Centre, The Royal Hospitals, Belfast, UK

Correspondence: MK O'Gallagher, Department of Ophthalmology, Eye and Ear Clinic Royal Victoria Hospital, Grosvenor Road, Belfast BT12 6BA, UK Tel: +44 (0)28 90632342; Fax: +44 (0)28 90634684 . E-mail: m.ogallagher@qub. ac.uk

Received: 29 February 2016 Accepted in revised form: 1 July 2016 Published online: 29 July 2016 
Jena, Germany) with laser interferometry. When calculating the lens power, an appropriate lens constant was used taking into account the biometry method. The cycloplegic refractive findings (performed by an experienced refractionist) were recorded at 3 months postoperatively to allow for the dissolution of sutures and the stabilisation of post-operative corneal astigmatism. The spherical and cylindrical measurements were converted to a spherical equivalent for the purposes of analysis.

\section{Two separate analyses were performed on the data}

(i) A change in policy in the department in 2001, following the Royal College of Ophthalmologists' Guidelines for adult cataract surgery (2001), ${ }^{4}$ resulted in a switch from the SRK-II formula to the SRK/T formula in paediatric cataract surgery; thus affording the opportunity to compare paediatric refractive outcome data for SRK-II (1996-2000) and SRK/T (2001-2008) formulae in an uncontrolled retrospective case series. Mean PE (ME), mean absolute error (MAE), SD and range were calculated for each group. A multiple regression analysis was performed. These statistical analyses were performed using the computer program R. ${ }^{5}$

(ii) The original biometry data from each case were then used, along with the dioptric power of the lens implanted, to calculate a predicted post-operative refraction for each of four adult lens prediction formulae (Hoffer $Q{ }^{6}$ Holladay $1,{ }^{7}$ SRK-II, $\left.{ }^{8} \mathrm{SRK} / \mathrm{T}^{9}\right)$. This was accomplished by using the software within the NIDEK US-1800.

The ME, SDs, $t$-test $P$-value, mean absolute error (MAE), and median absolute error (MedAE) were calculated for each formula. Mean square error of prediction (MSEP) was used to incorporate the variance and bias of the PEs.

$\operatorname{MSEP}=\frac{(n-1)}{n} s_{d}^{2}+d^{-2}$

where $s_{d}^{2}$ is the variance of the discrepancies and $d^{-2}$ is the square of the mean of the discrepancies (squared bias).

Lin's Concordance Correlation Coefficient (CCC) was also employed as a statistical measure of agreement between the predicted refractions of each formula and the actual post-operative refraction at 3 months. Statistical analyses were performed using the computer program R. ${ }^{5}$ Lin's CCC was obtained using function epi.ccc from R package epiR. ${ }^{10}$

Institutional board approval was obtained for this study from the Standards, Quality and Audit Department of the Belfast Health and Social Care Trust.

\section{Results}

Of the 46 patients (67 eyes) identified, case notes were available for 38 patients (55 eyes). Full data sets (including all the parameters above) were available for 27 patients (39 eyes), of whom 19 were male. For these 27 patients the mean age at the time of surgery was 3.8 years (range $0.25-7.25)$, and 7 patients (10 eyes) were aged 2 years or under.

The predicted refraction and actual refraction were available for 43 eyes. The MAE was 1.31 dioptres (D) (range $0.01-3.95 \mathrm{D}, \mathrm{SD} 0.98 \mathrm{D}$ ). More than $90 \%$ of PE were $<2.5 \mathrm{D}$.

\section{Analysis (i)}

The lens prediction of 15 eyes was calculated using SRK-II formula, and that of 27 eyes was calculated using SRK/T formula. It was not possible to ascertain which formula was used in one of the cases (one eye). The MAE using SRK-II was $1.38 \mathrm{D}$ and MedAE was $1.55 \mathrm{D}$ (range $0.28-2.47 \mathrm{D}, \mathrm{SD} 0.84 \mathrm{D}$ ). The MAE was smaller using SRK/T (1.30 D, MedAE 1.24, range 0.01-3.95 D, SD $1.08 \mathrm{D})$, but this difference is not statistically significant according to a two-sample $t$-test $(P=0.81)$ with $95 \%$ confidence limits $(-0.57,0.73)$.

The ME (all formulae) was $+0.21 \mathrm{D}$ (range: -3.25 to +3.95 D, SD 1.64 D). The ME was lower where the SRK/T formula was used $(-0.18 \mathrm{D}$, range -3.25 to $+3.95 \mathrm{D}, \mathrm{SD}$ $1.70 \mathrm{D})$ compared with where SRK-II was used (+ 0.96 D, range -2.47 to $+2.41 \mathrm{D}, \mathrm{SD} 1.33 \mathrm{D})$. A Student's $t$ - twosample test showed this difference to be statistically

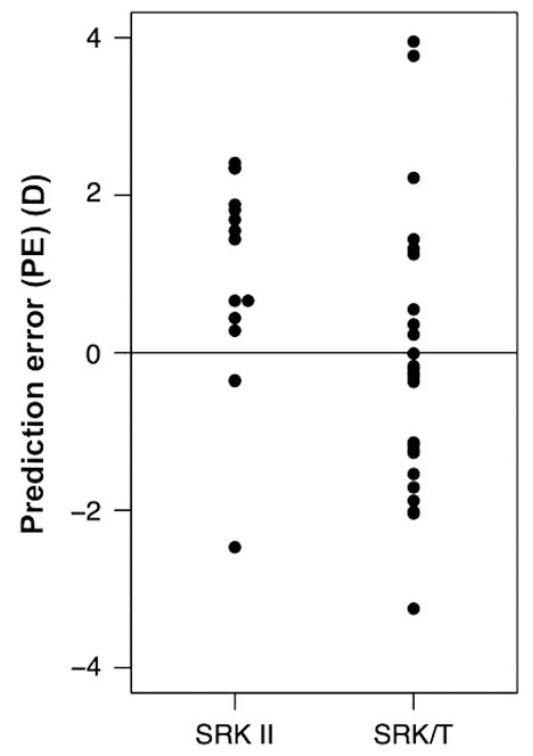

Figure 1 Box plot showing the spread of observed prediction errors for SRK-II and SRK/T. 
significant $(P=0.031)$, with $95 \%$ confidence limits 0.11-2.17. The spread of results for each formula can be seen graphically in the strip charts in Figure 1. The apparently larger variability of the SRK/T values arises from two or three values and is not statistically significant.

Neither age, nor axial length nor corneal curvature predicted the variability in the post-operative refraction. This was despite a tendency for older children and those with less short eyes to have a lower absolute PE.

\section{Analysis (ii)}

A graphical representation of the spread of PE for each of the four prediction formulae is shown in Figure 2.

A summary of the mean of each formula's PE, SD, 95\% confidence limits, MAE, and MedAE is given in Table 1. It can be seen from the data presented that the differences in MAE and MedAE are small between formulae but Hoffer $Q$ has the smallest MedAE.

The $t$-test $P$-value in Table 1 tests whether the ME differs from zero, and is therefore a test of bias. It appears therefore that there is statistical evidence that SRK-II and Hoffer $\mathrm{Q}$ are biased, having $P$-values of less than 0.05 ( 0.016 and 0.033 respectively). However, it should be noted that any bias, however small, would be statistically significant if the SD is sufficiently small. As a small SD is a good thing, it is possible that a good prediction formula would show a statistically significant bias.

Table 2 shows the MSEP for each prediction formula. From Table 2 the SRK/T and Holladay prediction formulae have the smallest MSEP, and the bias for SRK/T contributes the smallest percentage to its MSEP. Holladay 1 had the largest Lin's CCC (0.79), followed by SRK/T
(0.78) and Hoffer Q (0.78). The SRK-II predictions showed a much weaker correlation with the post-operative data with a Lin's CCC of 0.62 .

\section{Discussion}

We have found a high degree of variability in postoperative refraction data when both SRK-II and SRK/T had been used, though SRK/T showed greater accuracy on comparison of PE. In our second analysis, Holladay 1 showed accuracy which was approximate to that of SRK/T, while Hoffer Q showed precision but also bias (a tendency for the post-operative refraction to be more myopic than the prediction).

We set out to determine the accuracy of lens prediction formulae when used in a paediatric population. The degree of variability in results is similar to that reported in other series. ${ }^{11-16}$ SRK/T showed less bias in refractive outcomes than SRK-II, though this is based on small samples. Neely et al ${ }^{13}$ did not find a significant difference between SRK-T and SRK-II in their series.

Sources of inaccuracy and variability in paediatric lens power calculations are numerous. They may include measurement errors and errors in the assumptions made by prediction formulae, which were initially modelled on adult eyes. Obtaining accurate biometry measurements in children can be difficult. Most of our patients had axial length and keratometry measurements performed under anaesthesia. It can be difficult to obtain a true axial orientation with contact A-scan ultrasonography, with the resultant off-axis measurements resulting in inaccuracy. The same is true for keratometry readings. Applanation contact A-scan ultrasonography was used in the majority of our patients. A study of 22 paediatric eyes undergoing

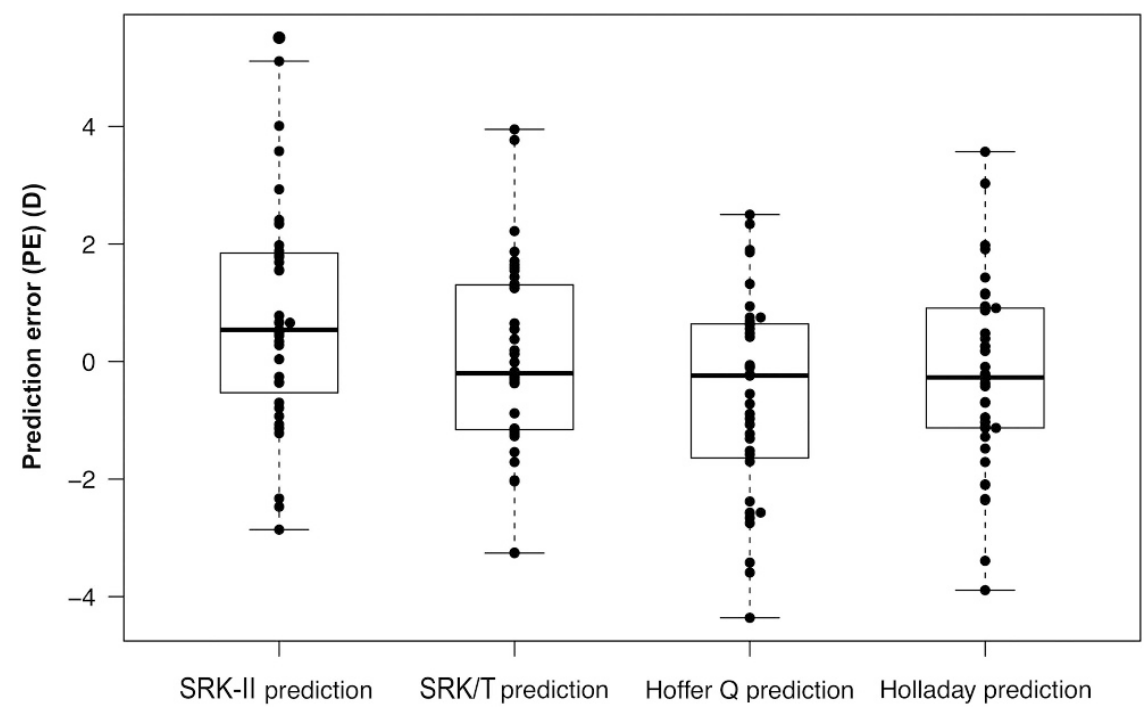

Figure 2 Strip chart showing prediction discrepancies for all formulae. 
Table 1 Prediction errors for each prediction formula

\begin{tabular}{lrcccccc}
\hline Prediction formula & $M E(D)$ & $S D$ & $t$-test P-value & Lower $95 \%$ confidence limit $(D)$ & Upper $95 \%$ confidence limit $(D)$ & MAE $(D)$ & MedAE $(D)$ \\
\hline Hoffer Q & -0.601 & 1.694 & 0.033 & -1.150 & -0.051 & 1.40 & 0.97 \\
Holladay I & -0.235 & 1.599 & 0.364 & -0.754 & 0.283 & 1.26 \\
SRK-II & 0.776 & 1.914 & 0.016 & 0.156 & 0.397 & 1.03 \\
SRK/T & 0.038 & 1.634 & 0.886 & -0.492 & 0.567 & 1.58 & 1.14 \\
\hline
\end{tabular}

Table 2 Squared bias and mean square error of prediction (MSEP) by formula

\begin{tabular}{lcccr}
\hline Prediction formula & Squared bias & Variance of discrepancies & MSEP & Squared bias as a percentage of MSEP \\
\hline Hoffer Q & 0.3606 & 2.869 & 3.156 & 11.43 \\
Holladay & 0.0554 & 2.557 & 2.547 & 2.18 \\
SRK-II & 0.6024 & 3.662 & 4.171 & 14.44 \\
SRK/T & 0.0014 & 2.671 & 2.604 & 0.05 \\
\hline
\end{tabular}

cataract surgery has suggested that immersion A-scan ultrasonography is more accurate than contact A-scan, ${ }^{17}$ although it is acknowledged that immersion ultrasonography requires skill and experience ${ }^{18}$ and is not available in many units in the United Kingdom. ${ }^{19}$ When using A-scan ultrasound, the speed of the ultrasound waves is affected by the density of the material through which it is passing. Very dense cataracts may influence the final measurement to a greater extent in shorter eyes as the lens makes up a relatively greater proportion of the axial length. Further controversy exists on whether the increased density of the paediatric vitreous should be taken into account in the ultrasound velocity used in calculating the axial length. ${ }^{20}$

The magnitude of deviation introduced by measurement errors in shorter eyes may be greater than in those of average length. Eibschitz-Tsimhoni et al ${ }^{21}$ noted that the sensitivity of IOL power calculations to axial length measurement error is increased at 4-14 D per millimetre of error in axial length in children as compared with 3-4 D per millimetre error in axial length in adults. They found a sensitivity to keratometry error of $0.8-1.3$ $\mathrm{D}$ per (keratometry) dioptre in both adults and children alike.

The prediction formulae are another possible source of variability and we sought to examine this. We showed that SRK/T had greater precision than SRK-II based on observed outcomes. Some studies on paediatric populations have shown no difference between SRK/T and SRK-II formulae. ${ }^{3,13}$ However, a theoretical analysis of prediction formulae including SRK/T and SRK-II for biometry data in the paediatric range showed differences in predicted implant powers with different formulae. ${ }^{1}$ Amongst adult patients, SRK/T has been shown to be superior to the extent that the Royal College of
Ophthalmologists in the United Kingdom in its 2004 guidelines (updated 2007) 22 has advised that SRK-II should be regarded as obsolete and SRK-T be used in its place. Meanwhile, SRK-II remains in use in some centres, with adequate results reported ${ }^{23}$ (Morales, 2009, unpublished data presented at WCPOS Conference, Barcelona).

Investigators who have examined the post-operative refractive results in paediatric cataract surgery have found a high degree of variability in results using SRK-II and SRK/T. ${ }^{11-13}$ Mezer et $a l^{15}$ compared the predictions of five formulae (SRK, SRK-II, SRK/T, Holladay 1 and Hoffer Q) in 206 primary and secondary lens implantations in children and found the results of all the formulae to be unsatisfactory. Eibschitz-Tsimhoni et $a l^{24}$ found Holladay 1, Haigis and SRK/T to be comparable using theoretical combinations of biometry data in the paediatric range, although SRK/T was not as accurate at high keratometry values. In the Infant Aphakia Treatment Study, the Hoffer Q, Holladay 1, Holladay 2, SRK-II, and $\mathrm{SRK} / \mathrm{T}$ formulae were used to predict refractive outcomes in 43 eyes operated on at an average age of 2.5 months with an average axial length of $18.1 \pm 1.1 \mathrm{~mm}$. The outcomes were similar to our findings with SRK/T and Holladay 1 showed the best results with mean absolute PEs of $1.4 \pm 1.3 \mathrm{D}$ and $1.7 \pm 1.1 \mathrm{D}$, respectively. ${ }^{25}$ Nihilani and VanderVeen ${ }^{26}$ compared PEs of SRK-II, SRK/T, Holladay 1 and Hoffer Q formulae in 135 eyes of 96 paediatric patients and found the PEs of all formulae to be comparable, although Hoffer Q was most accurate for the highest number of eyes. Neely et al, ${ }^{13}$ analysing the outcomes of 101 actual paediatric cataract operations, found no observed difference between SRK-II and SRK/T. They performed a theoretical comparison of lens prediction formulae using the actual biometry data and refractive 
outcomes to decide on an ideal IOL power.

This method involves significant assumptions of the effective lens power of the IOL, which we have tried to avoid with our calculations. They found no significant difference between formulae, but did note, however, SRK-II was unreliable at the extremes of lens power values, and a positive bias in the results of Hoffer $Q$.

The findings of this study and others on the accuracy of lens power calculations in paediatric cataract surgery have shown accuracy of prediction to be less than that which could be expected in adult populations. It is recognised that the biometric data involved, and in particular axial lengths, are at the extremes of the normal range of adult eyes. ${ }^{22}$ Furthermore, it could be argued that congenital and developmental cataracts represent a subgroup of the normal paediatric population and deviations from normal ophthalmic anatomy may be expected.

Clearly the accuracy of regression formulae, such as SRK-II, will be sub-optimal as the data set on which the regression analysis was performed contained very few short eyes. ${ }^{8}$ Theoretical formulae could reasonably be expected to perform better in this regard, being based on optical principles. However, this assumes that all aspects of children's eyes are smaller than adults' eyes in equal proportions, so that the optical geometry is similar. This is not known and may not be true, especially with regard to aspects having a major role in the third generation formulae such as post-operative anterior chamber depth, corneal height and width, retinal thickness and corneal refractive index. In the SRK/T formula, the optimum values for these variables were empirically derived with reference to a data set of adult eyes. ${ }^{9}$ In paediatric patients it is common to aim, not for emmetropia, but for a degree of hypermetropia that takes into account the expected emmetropisation with age and the degree of anisometropia that will occur relative to the refractive state of the fellow eye. Children undergoing surgery in our study had their refractive outcome matched for age in keeping with the findings of Flitcroft et al. ${ }^{27}$ Infants less than 12 months of age had a refractive goal of $+6 \mathrm{D}$, decreasing to $+3 \mathrm{D}$ at 3 years of age and emmetropia at 8 years of age. Given that the prediction formulae are usually used and originally designed to aim for emmetropia (or close to it), it is possible that they are less accurately applicable to the higher refractive goals employed in paediatric cataract surgery. 8,9 Against this argument are Eibschitz-Tsimhoni's ${ }^{24}$ findings of similar errors when aiming for $+6 \mathrm{D}$ and emmetropia in prediction calculations using theoretical paediatric biometry values. Of course, considerable debate exists over the degree of emmetropisation that should be expected and what the targeted refraction should be if not emmetropia. ${ }^{28}$
The major limitation of this study was the small number of eyes included, making statistical analysis and interpretation difficult. We recognise the potential bias of including both eyes in bilateral cases but are keen that this study reflects practice in the real world. Furthermore, being a retrospective study, there are many sources of potential bias for which the analysis was unable to control, for example, minor variations in surgical technique between surgeons (of which there were three).

We recognise that the comparison between SRK/T and SRK-II was uncontrolled and consecutive (as opposed to being in parallel), but nevertheless feel that our data are worthy of report. Of note, SRK-II was not used in any child under 2 years of age, because it had been replaced by SRK/T in this department before it became accepted practice to implant IOLs in younger children. The sample sizes were small and also unequal; there was a larger range of values for SRK/T resulting in a larger SD but this was more than compensated for by the larger number of values when compared to SRK-II. Some case notes were not available for review and, given the retrospective nature of the study, some of the information required had not been recorded in the notes. A larger, prospective study would be valuable in further investigating this subject, however even tertiary referral centres treat only small numbers of paediatric cataracts per year making the organisation of large studies difficult.

Given the difficulties described here in accurately predicting post-operative refraction in paediatric cataract surgery with primary IOL implantation, it could be argued that there is a role for a lens power calculation formula designed for use in the paediatric population. For such a formula to be developed, however, it must be remembered that the differences between children's and adults eyes may not be confined solely to dimensions. As has been noted in paediatric practice in other branches of medicine and surgery, 'children are not small adults'. ${ }^{29}$

\section{Summary}

What was known before

- Lens power prediction error shows a higher degree of variability in paediatric populations.

What this study adds

- SRK/T was more accurate than SRK-II in this paediatric population. Hoffer Q may be more accurate on the basis of a theoretical analysis.

\section{Conflict of interest}

The authors declare no conflict of interest. 


\section{Author contributions}

Design of the study: MOG, MP, and EMcL; conduct of the study: MOG, MAL, MP, and EMcL; preparation of manuscript: MOG; review and approval of manuscript: $\mathrm{CM}$ and $\mathrm{GMcG}$.

\section{Acknowledgements}

This work has previously been presented as a poster at the World Congress of Paediatric Ophthalmology and Strabismus, Barcelona, Spain, September 2009, and at the Annual Congress of the Royal College of Ophthalmologists, Liverpool, UK, 2010

\section{References}

1 Eibschitz-Tsimhoni M, Archer SM, Del Monte MA. Intraocular lens power calculations in children. Surv Ophthalmol 2007; 52: 474-482.

2 Solebo AL, Russell-Eggitt I, Nischal KK, Moore AT, Cumberland P, Rahi JS et al. on behalf of the British Isles Congenital Cataract Interest Group. Cataract surgery and primary intraocular lens implantation in children $\leq 2$ years old in the UK and Ireland. Br J Ophthalmol 2009; 93: 1495-1498.

3 Andreo LK, Wilson ME, Saunders RA. Predictive value of regression and theoretical IOL formulas in pediatric intraocular lens implantation. J Pediatr Ophthalmol Strabismus 1997; 34: 240-243.

4 The Royal College of Ophthalmologists. Cataract Surgery Guidelines, 2nd ed. The Royal College of Ophthalmologists: London, UK, 2010.

5 R Development Core Team, R. A Language and Environment for Statistical Computing. R Foundation for Statistical Computing: Vienna, Austria, 2011.

6 Hoffer KJ. The Hoffer Q formula: a comparison of theoretic and regression formulas. J Cataract Refract Surg 1993; 19: 700-712.

7 Holladay JT, Prager TC, Chandler TY, Musgrove KH, Lewis JW, Ruiz RS. A three-part system for refining intraocular lens power calculations. J Cataract Refract Surg 1988; 14: 17-24.

8 Sanders DR, Retzlaff J. Comparison of the SRK-II formula and other second generation formulas. J Cataract Refract Surg 1988; 14: 136-141.

9 Retzlaff JA, Sanders DR, Kraff MC. Development of the SRK/T intraocular lens implant power calculation formula. J Cataract Refract Surg 1990; 16: 333-340; erratum, 1990; 16(4): 528.

10 Stevenson $M$ with contributions from Nunes $\mathrm{T}$, Sanchez J, Thornton R, epiR: Functions for analysing epidemiological data, $\mathrm{R}$ package version 0.9-32 http:/ /CRAN.R-project.org/ package $=$ epiR, 2011.

11 Barry J-S, Ewings P, Gibbon C, Quinn AG. Refractive outcomes after cataract surgery with primary lens implantation in infants. Br J Ophthalmol 2006; 90: 1386-1389.

12 Tromans C, Haigh PM, Biswas S, Lloyd IC. Accuracy of intraocular lens power calculations in pediatric cataract surgery. Br J Ophthalmol 2001; 85: 939-941.
13 Neely DE, Plager DA, Borger SM, Golub RL. Accuracy of intraocular lens calculations in infants and children undergoing cataract surgery. J AAPOS 2005; 9: 160-165.

14 Astler WF, Ingram AD, Isaza GM, Echeverri P. Pediatric pseudophakia: analysis of intraocular lens power and myopic shift. Clin Experiment Ophthalmol 2007; 35: 244-251.

15 Mezer E, Rootman DS, Abdolell M, Levin AV. Early postoperative refractive outcomes of pediatric lens implantation. J Cataract Refract Surg 2004; 30: 603-610.

16 Hoevenaars NED, Polling JR, Wolfs RCW. Prediction error and myopic shift after intraocular lens implantation in paediatric cataract patients. Br J Ophthalmol 2011; 95: 1082-1085.

17 Trivedi RH, Wilson ME. Prediction error after pediatric cataract surgery with intraocular lens implantation: Contact versus immersion A-scan biometry. J Cataract Refract Surg 2011; 37: 501-505.

18 Trivedi RH, Wilson ME. Axial Length Measurements by Contact and Immersion Techniques in Pediatric Eyes with Cataract. Ophthalmology 2011; 118: 498-502.

19 Gale RP, Saha N, Johnston RL. National Biometry Audit II. Eye (Lond) 2006; 20: 25-28.

20 Mehdizadeh M. Effect of axial length and keratometry measurement error on intraocular lens implant power prediction formulas in pediatric patients. J AAPOS 2008; 12: 425.

21 Eibschitz-Tsimhoni M, Tsimhoni O, Archer SM, Del Monte MA. Effect of axial length and keratometry measurement error on intraocular lens implant power predictive formulas in pediatric patients. J AAPOS 2008; 12: 173-176.

22 The Royal College of Ophthalmologists. Cataract Surgery Guidelines, 1st ed. The Royal College of Ophthalmologists: London, UK, 2004, updated 2007.

23 Ram J, Gupta N, Sukhija JS, Chaudhary M, Verma N, Kumar S et al. Outcome of cataract surgery with primary intraocular lens implantation in children. Br J Ophthalmol 2011; 95(8): 1086-1090.

24 Eibschitz-Tsimhoni M, Tsimhoni O, Archer SM, Del Monte MA. Discrepancies between intraocular lens implant power prediction formulas in pediatric patients. Ophthalmology 2007; 114: 383-386.

25 Vanderveen DK, Trivedi RH, Nizam A, Lynn MJ, Lambert SR. Infant Aphakia Treatment Study Group. Predictability of intraocular lens power calculation formulae in infantile eyes with unilateral congenital cataract: results from the Infant Aphakia Treatment Study. Am J Ophthalmol 2013; 156: 1252-1260.

26 Nihilani BR, VanderVeen DK. Comparison of intraocular lens power calculation formulae in pediatric eyes. Ophthalmology 2010; 117: 1493-1499.

27 Flitcroft DI, Knight-Nanan D, Bowell R, Lanigan B, O'Keefe M. Intraocular lenses in children: changes in axial length, corneal curvature, and refraction. Br J Ophthalmol 1999; 83: 265-269.

28 Nischal KK, Solebo L, Russell-Eggitt I. Paediatric IOL implantation and postoperative refractive state: what role do study methodology and surgical technique play? Br J Ophthalmol 2010; 94: 529-531.

29 Bachrach LK. Bare bones fact-children are not small adults. N Engl J Med 2004; 351: 924-926. 